\title{
The Potential of Atomistic Simulations and the Knowledgebase of Interatomic Models
}

\author{
E.B. Tadmor, R.S. Elliott, J.P. Sethna, R.E. Miller, and C.A. Becker
}

Atomistic simulations are playing an increasingly important role in realistic scientific and industrial applications in many areas including advanced materials design, drug design, renewable energy, and nanotechnology. The predictive capability of these approaches hinges on the accuracy of the model used to describe atomic interactions. Modern models are optimized to reproduce experimental values and electronic structure estimates for the forces and energies of representative atomic configurations deemed important for the problem of interest. However, no standardized approach exists for estimating the accuracy of interatomic models. In addition, a lack of standardization in the programming interface of interatomic models and the lack of a systematic infrastructure for archiving and retrieving them make it difficult to use existing models for new applications and to reproduce published results. These limitations prevent the field of atomistic modeling from realizing its true scientific and technological potential.

These limitations are being addressed by a four-year project initiated in October 2009 as part of the National Science Foundation Cyber-Enabled Discovery and Innovation program, led by the authors of this article, called the Knowledgebase of Interatomic Models (KIM). The project consists of two stages, each of which would constitute a significant contribution to atomistic computational materials science. In the first stage, an open-source online infrastructure consisting of a web por- tal, repository, and processing pipeline is being developed at http://openKIM .org. The repository will contain interatomic models, simulation codes that test the predictions of these models (referred to as "tests" for short), and reference data obtained from first principles calculations and experiments. Users will be able to freely upload and download models and tests and to search the content of the site. When a new model is uploaded, a processing pipeline will automatically couple it with all tests already stored in the repository, generating new predictions that will be stored in the repository. Similarly new tests will be coupled with all models on the site to generate new predictions. In this way a great deal of information on the accuracy of interatomic models will be gathered over time. Development of the infrastructure described above will also involve the establishment, in collaboration with the National Institute of Standards and Technology and the research community, of terminology dictionaries, testing/benchmarking procedures, and application programming interface (API) standards for atomistic simulations. In particular, the KIM API will allow any interatomic model to work seamlessly with any atomistic simulation code.

The second stage of the KIM project will focus on developing a framework to evaluate the transferability and precision of interatomic models. Transferability refers to the ability of a model to provide accurate predictions for properties that it was not fitted to reproduce. Precision refers to the error bars associ- ated with the prediction of a particular type of model for a given property. The goal is to develop automatic guidelines for the selection of appropriate models for a given scientific or technological application. If successful, this effort has the potential to revolutionize the manner in which interatomic models are used in technological applications.

The KIM project is being carried out in close collaboration with members of the atomistic simulation community. The KIM Inaugural Workshop was held in February 2011 in San Diego, California, during the TMS 2011 Annual Meeting. Participants included many key interatomic model and molecular dynamics code developers. At the workshop a Requirements Document (RD) for KIM was created. The RD will serve as a living document that will accompany the KIM project as it develops. This document will be placed on-line at http :/lopenKIM.org, where visitors will be able to provide feedback. We invite the entire atomistic simulation community to participate in the KIM project and to contribute to the standardization efforts underway.

E.B. Tadmor, professor, and R.S. Elliott, associate professor, are with the Department of Aerospace Engineering and Mechanics, University of Minnesota, Minneapolis, MN 55455, USA; J.P. Sethna, professor, is with the Department of Physics, Cornell University, Ithaca, NY; R.E. Miller, professor, is with the Department of Mechanical and Aerospace Engineering, Carleton University, Ottawa, Canada; and C.A. Becker, materials research engineer, is with the Metallurgy Division, National Institute of Standards and Technology, Gaithersburg, MD. Dr. Tadmor can be reached at tadmor@aem.umn.edu.

\section{E.B. Tadmor, R.S. Elliott, and C.A. Becker are TMS Members!}

To read more about them, turn to page 8. To join TMS, visit www.tms.org/Society/Membership.aspx.

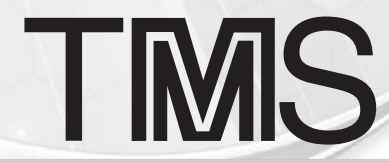

